\title{
Hacia la construcción de un modelo de liderazgo intergeneracional
}

\author{
Rocío Moldes \\ rocio.modes@universidadeuropea.es \\ Universidad Europea \\ Fátima Gómez \\ fatima.gomez@universidadeuropea.es \\ Universidad Europea
}

Resumen: Este artículo se inserta en el estudio de los nuevos estilos de "liderazgo" contemporáneo, estrechamente vinculado a los planteamientos teóricos del compromiso. El objetivo es, en primer lugar, caracterizar, a partir de diez categorías de análisis, las diferencias y similitudes en el estilo de dirección, según sea la generación del líder, percibidas por sus empleados ${ }^{1}$. Posteriormente y basándonos en los resultados del análisis de los discursos, obtenidos mediante la técnica del grupo de discusión, proponemos la construcción de un modelo del liderazgo intergeneracional, enmarcada en dos competencias transversales —inteligencia emocional y perspectiva generacional一, como herramienta para aprovechar el talento intergeneracional.

Palabras clave: compromiso, competencias, retención del talento, liderazgo colaborativo, liderazgo intergeneracional, diversidad generacional.

1 El contenido del artículo se basa en los resultados obtenidos en el proyecto de investigación titulado: "Liderazgo intergeneracional: análisis de los comportamientos directivos según la generación de los managers y cómo desarrollar su liderazgo para la gestión de equipos intergeneracionales", realizado por el Observatorio Generación y Talento en colaboración con la consultoría Tatum y las profesoras Rocío Moldes y Fátima Gómez de la Universidad Europea. El trabajo de campo fue realizado entre septiembre de 2017 y marzo de 2018. 


\begin{abstract}
This article studies new styles of contemporary "leadership", an area closely linked to theoretical approaches to commitment. The main objective is to use ten analysis categories to characterize differences and similarities in management style in terms of the leader's generation as perceived by their employees. The study then analyses the opinions and points of view expressed in the focus group and proposes the construction of a model of intergenerational leadership, comprising the two transversal competences of emotional intelligence and generational perspective, as a tool for getting the best out of intergenerational talent
\end{abstract}

Keywords: commitment, skills, retaining talent, collaborative leadership, intergenerational leadership, generational diversity. 


\section{Introducción}

La afirmación de que las "personas son el activo más importante de la empresa" (Drucker, 1996) se ha convertido durante las últimas décadas en una máxima indiscutible en el ámbito de la dirección de personas. Las causas que han conducido a considerar a los empleados como el recurso estratégico por excelencia hay que buscarlas en las transformaciones acontecidas en la llamada "sociedad del conocimiento". En su gran mayoría el valor de las empresas deriva de activos intangibles (Cheese, 2008), tales como el conocimiento del mercado, las relaciones con clientes y proveedores, el valor de la marca, la reputación empresarial y, por supuesto, las habilidades de los trabajadores. Todos estos activos están basados en el talento humano. La idea es que, en un mercado cada vez más abierto y competitivo por el uso de sistemas de transporte eficientes y baratos y las tecnologías de la información, restan pocos recursos tradicionales que no se puedan copiar o sustituir.

En la mayoría de los casos el único recurso que puede garantizar una ventaja competitiva sostenible tiene un carácter intangible: las competencias y la implicación de los empleados en la organización. Dada la diversidad demográfica y de capital cultural, gestionar dicha diversidad ha dejado de ser una ventaja competitiva, un valor diferencial, para convertirse en un requisito en la dirección de personas en las organizaciones (Dychtwald et al., 2007). Este hecho responde básicamente a que las estructuras homogéneas han dejado de tener sentido en las sociedades contemporáneas. Asimismo, la gestión de la diversidad en la toma de decisiones no responde solo a razones éticas y legales, sino también a los beneficios empresariales que conlleva. Esto es así porque cualitativamente la evolución de las políticas de diversidad es medible mediante las encuestas de clima laboral, las cuales evidencian que la satisfacción y el compromiso incrementan la productividad (CEDE, 2015). Desde el punto de vista cuantitativo, se trata de establecer indicadores claros y contrastables que permitan medir el objetivo que persigue la iniciativa implantada, como, por ejemplo, ampliar la base de clientes, facilitar el acceso a los mercados, etc. Asimismo, la diversidad mejora la imagen corporativa y la reputación de las empresas (Moldes, 2010).

El hecho es que, en el competitivo contexto profesional del siglo xxi, la exigencia de trabajo en equipo convierte en muy relevantes a aquellas personas capaces de transformar el potencial individual de sus colaboradores en talento colectivo (Marí, 2017). El trabajo de un directivo suele ser difícil y costoso, ya que consiste en obtener las cosas por medio de otros. Un buen líder tiene que intentar "sacar" lo mejor de cada persona, no "colocar" dentro de ella lo que esta fuera. 
Nuestra propuesta del liderazgo intergeneracional se enmarca en este contexto de transformación constante en el que la figura del líder se ha visto progresivamente desplazada por la competencia del liderazgo (Pastor, 2009).

Este modelo se halla estrechamente vinculado con dos competencias transversales: la inteligencia emocional y la perspectiva generacional. Ambas categorías se presentan actualmente como pilares en el desarrollo y aplicación del liderazgo colaborativo y el modelo de compromiso, que pasamos a detallar en el siguiente epígrafe.

\section{Liderazgo colaborativo y modelo de compromiso}

Los primeros esfuerzos por sistematizar el liderazgo se enfocaron en definir las características personales de los líderes. Buscaban un atributo que distinguiera, de manera clara y consistente, a los líderes de los seguidores. Los estudios posteriores se centraron en determinar los rasgos distintivos de un líder efectivo de otro que no lo es, pero el resultado fue igual de infructuoso. Solo logró determinarse que la eficacia del líder no depende de unos rasgos particulares, sino más bien de cómo los rasgos del líder se adecúan a los requisitos de la situación. Una tercera etapa de análisis, enmarcada más en el concepto de competencias (Maella, 2008) que en el de habilidades técnicas, se centró en la capacidad del líder para lograr el compromiso de los colaboradores.

Alineado con esta concepción del liderazgo, el modelo del compromiso en las organizaciones predica que este es uno de los factores centrales para retener el talento. Se relaciona directamente con el del liderazgo en el sentido de que son los líderes quienes deben crear entornos de relación capaces de incentivar e incrementar el compromiso entre sus colaboradores. Así, paralelamente al desarrollo de los estudios sobre liderazgo se desarrolla el modelo del compromiso, dentro del cual el papel del líder se va configurando como esencial. Por tanto, un líder deberá cumplir tres tareas fundamentales: adquirir y dar información (a fin de desarrollar su función principal: tomar decisiones), desarrollar y mantener relaciones, e influir a otros.

Los elementos comunes a toda persona con capacidad de liderazgo se pueden sintetizar en los siguientes: establecer una dirección, alinear a los colaboradores con la dirección marcada e inspirar a los colaboradores (Moldes, 2012).

La competitividad de las empresas va a estar directamente relacionada con la capacidad que estas tengan para atraer, retener y desarrollar el talento. Sin embargo, en los entornos VUCA y con la incorporación a las organizaciones de la generación Y (millennials) y Z, nos encontramos en un nuevo entorno organi- 
zacional donde la fidelidad de los profesionales hacia sus empresas es cada vez menor, con lo cual parece difícil comprometer al capital humano a largo plazo.

El compromiso como factor central en la retención del talento y la forma de gestionarlo comienzan a estudiarse principalmente en la década de 1990. Uno de los principales enfoques explicativos más aceptados ha sido el desarrollado por Meyer y Allen en la década de 1990. Estos autores defienden la existencia de tres tipos de compromiso: de continuación, normativo y afectivo (Allen y Meyer, 1990; Meyer y Allen, 1991).

El compromiso de continuación, según estos autores, se basa en la existencia de unas circunstancias que hacen sentir al trabajador que debe seguir en la empresa. Estas pueden ser el coste de abandono, la ausencia de alternativas interesantes para el empleado y el tiempo o los esfuerzos invertidos en la empresa. Por lo tanto, se mueve por el interés (Gavilán, Avelló y Fernández-Lores, 2013).

El compromiso normativo, propio de las sociedades industriales y burocratizadas, se basa en la interiorización de las normas que obligan al empleado a actuar de tal manera que facilite a la organización alcanzar sus objetivos. Finalmente, el compromiso afectivo o "actitudinal" fue identificado por Kanter (1968) como un compromiso de "cohesión", de unión emocional y afectiva que va del individuo al grupo. En este caso, del empleado a la empresa a la que se siente unido por los lazos de pertenencia afectiva. El modelo de compromiso afectivo se basa en "la experiencia" que el empleado tiene con la organización a la que pertenece. Esta se puede clasificar en tres dimensiones (Ambler y Barrow, 1996):

- Sensorial/estética: conjunto de estímulos sensoriales que suministra la marca a través del espacio físico donde se realiza el trabajo.

- Intelectual: hace referencia a los valores y a lo que supone para el empleado el acercamiento, conocimiento y su interiorización.

+ Emocional o de entretenimiento: hace referencia al disfrute del trabajo. Se relaciona directamente con las vivencias del desempeño en el trabajo.

Basándose en estas dimensiones, las investigadoras Gavilán, Avelló y Fernández (2013) realizaron un estudio empírico para comprobar la experiencia con la marca-empresa y su impacto con el compromiso afectivo ${ }^{2}$. A partir de esta investigación, las autoras, junto con la consultoría Tatum ${ }^{3}$, desarrollaron un modelo descriptivo, explicativo y predictivo de evaluación del compromiso que las perso-

2 Los resultados de esta investigación se presentan en el artículo: "Employer branding, la experiencia social de la marca y sus efectos sobre el compromiso afectivo". aDResearch ESIC, n 7, volumen 7, junio 2013.

3 Tatum (Improving people $\&$ sales) ha desarrollado y validado un modelo descriptivo, explicativo y predictivo que evalúa tanto el compromiso afectivo como sus antecedentes y sus consecuentes (comportamientos). Utiliza el término compromiso para referirse únicamente a la dimensión afectiva. El Modelo TEEC. Véase: informetatum-barometro-del-compromiso-en-epana-2015/ 
nas generan a partir de la experiencia "vivida” en la organización. Se identificaron cinco variables principales determinantes del compromiso en la organización: cultura, función, equipo, relación y jefe/a. De ellas, el estilo de liderazgo es una de las dimensiones clave para la creación del vínculo en las organizaciones, junto con el reconocimiento de logros, la gestión integral de las personas y el apoyo recibido.

Los diferentes estudios sobre el compromiso en las organizaciones establecen que el liderazgo es una de las claves. Son los líderes quienes a través de un liderazgo colaborativo pueden promover y mantener el compromiso de su equipo reteniendo el talento de los colaboradores.

En resumen, ser capaz de incentivar el compromiso de los empleados con la organización constituye una competencia central del líder. Este debe saber gestionar las diferentes dimensiones (esencialmente la "intelectual" y la "emocional") de la experiencia del empleado, la cual, como se ha visto en los diferentes estudios, influye directamente en la creación de un compromiso afectivo con la organización. La implantación de un modelo de compromiso debe acompañarse de un liderazgo de tipo colaborativo que haga hincapié en los procesos relacionales y la gestión emocional.

Así, el liderazgo colaborativo consolida la tendencia según la cual los estudios sobre liderazgo contemporáneo no pueden centrarse en las características de un individuo, como dictaba el enfoque tradicional, sino en la construcción de una competencia organizacional.

El nuevo liderazgo solo se desarrolla mediante procesos relacionales. Este es su escenario, de forma que la función de líder no la desempeñará el empleado más brillante, sino el que tenga más capacidad para hacer brillar al resto (Moldes, 2010).

En el contexto actual, la gestión de personas se marca como objetivo prioritario obtener el compromiso recíproco entre los empleados y la organización mediante la implantación de estilos de dirección basados en la participación (Goleman y Drucker, 2018). Para ello, se hace imprescindible el liderazgo organizacional; solo así se pueden integrar conceptos como innovación, creatividad, inteligencia emocional o habilidades comunicativas, que constituyen la piedra angular sobre la que "presentarse" en el ámbito laboral.

La herramienta clave de la que se servirá este modelo de dirección de equipos es un sistema interno de comunicación basado en la gestión emocional. Este enfoque ha propiciado el desarrollo y auge de una prolífica literatura en torno a las nociones de "feelings management" (Conger, 1993), "storytelling management" (Salmon, 2009) y, especialmente, "inteligencia emocional" (Goleman,1995). Todos ellos orientados a conseguir la deseada comunicación eficaz. 
La idea es que la emoción es un lenguaje universal que permite "controlar" mejor los tres grandes rasgos (Moldes, 2012) que definen las plantillas contemporáneas, a saber:

- El uso de las tecnologías, la importancia de las redes (sistema dinámico y cooperativo) y, sobre todo, su capacidad para invalidar la comunicación vertical.

- La cualificación de los empleados; los mensajes claros funcionan mejor que la persuasión.

- La diversidad de los equipos; entre plantillas diversas el objetivo principal del jefe inmediato debe ser minimizar las diferencias en la interpretación de los mensajes.

Así, el liderazgo colaborativo (Álvarez de Mon, 2009) se define como aquel que contribuye activamente a la implicación de los miembros del equipo, abandona la jerarquía y se basa en la formulación de preguntas, reconociendo la dificultad de presentar respuestas. El objetivo principal del jefe inmediato debe ser minimizar las diferencias en la interpretación de los mensajes. El nuevo modelo está caracterizado, en primer lugar, por un cambio terminológico: del líder al liderazgo. Este cambio consolida la tendencia, iniciada con el liderazgo transaccional, según la cual no se trata de las características de un individuo o de un rol centrado sobre una persona, sino de una competencia organizacional.

Cabe señalar, como cierre de este apartado, que trabajos recientes (RedondoSama, 2020) ilustran cómo el ejercicio del liderazgo dialógico apoya la fortaleza de los principios democráticos y la calidad del empleo en las organizaciones en las que está implantado.

\section{El liderazgo intergeneracional: una aproximación cualitativa al contexto español}

En el panorama internacional los estudios sobre liderazgo intergeneracional han ido constatando la importancia de identificar las diferentes vinculaciones entre generaciones y liderazgo con vistas a la consecución de los objetivos del equipo y el desarrollo del talento dentro de la organización.

Entre los trabajos pioneros cabe destacar el titulado Retención del talento (Dychtwald, Erickson y Morison, 2007), donde los autores señalan, hace casi tres lustros, la importancia de gestionar la diversidad generacional y la necesidad de atender a las diferencias en las expectativas y estilos de trabajo de las diferentes generaciones. Los cambios demográficos y culturales que afectan al mercado laboral exigen una gestión adaptada a cada segmento de trabajadores 
para responder a la escasez de habilidades y talento derivada de la jubilación de las generaciones sénior (veteranos y baby boomers) y a la incorporación de las generaciones de los llamados "nativos digitales" (Prensky, 2001).

Durante la primera década del presente siglo irán apareciendo diferentes publicaciones centradas en debatir las diferencias generacionales como factores que, de forma directa, afectan a los niveles de productividad y satisfacción en el trabajo, el estilo de gestión y la motivación personal (Warner y León, 2010).

Más recientemente, destacan en el contexto anglosajón y americano estudios empíricos como el informe titulado "Building leaders for the next decade" (2017). En este estudio, que se enmarca dentro del proyecto Generations ${ }^{4}$, se exploran aspectos tales como la percepción de las diferentes generaciones sobre el liderazgo, las barreras más importantes a la hora de acceder a puestos de liderazgo, o la concepción sobre la manera de ejercerlo. Lo que constata el trabajo es que todas estas cuestiones son diferentes en función de las generaciones, que su significado varía sustancialmente al cruzar la variable de generación con la de género y que el interés por los puestos de liderazgo presenta grandes diferencias según los países a los que hagamos referencia.

Parece evidente que los efectos demográficos, combinados con la revolución tecnológica, constituyen el gran desafío de la gestión de personas en el contexto de la cuarta revolución industrial.

En España, hasta el momento, existen dos estudios pioneros sobre la gestión de la diversidad generacional en las empresas.

El primero de ellos se titula: "Diagnóstico de la diversidad generacional. Análisis del talento intergeneracional en las empresas". Lo llevaron a cabo, entre septiembre de 2015 y noviembre de 2016, el Observatorio Generación y Talento y la Universidad Pontificia de Comillas ICAI-ICADE. Su principal aportación consistió en constatar la existencia de cinco generaciones diferentes caracterizadas según sus estilos de trabajo y expectativas vitales cuya gestión era, además de un reto, un imperativo. De forma que en el actual mercado de trabajo español pueden "convivir" entre tres y cinco generaciones en una misma empresa: veteranos, baby boomers, generación $\mathrm{X}$, generación $\mathrm{Y}$, generación $\mathrm{Z}^{6}$.

\footnotetext{
4 Serie de estudios llevados a cabo por Universum, INSEAD Emerging Markets Institute, The HEAD Foundation y el MIT Leadership Center. Se trata de diferentes investigaciones sobre cómo piensan las generaciones "globales" acerca de sus empleadores y lugares de trabajo. Con especial atención a su definición de liderazgo. Los resultados se basan en una encuesta anual sobre una muestra de 18.000 estudiantes y profesionales llevada a cabo en diecinueve países.

5 El enlace al informe ejecutivo puede encontrarse en: $\langle$ https://www.generacciona.org/generaciones.pdf $>$.

6 El estudio, de carácter cuantitativo, está basado en los resultados obtenidos en 3.697 encuestas enviadas a quince organizaciones. Las diferentes generaciones se clasifican según el siguiente criterio: sénior o veteranos (nacidos antes de 1955); baby boomers (1956-1970); generación X (1971-1981); generación Y (1982-1992); generación Z (1993-2008).
} 
Cualquier empresa que aspire a operar en un mundo global y diverso tendrá, pues, como objetivo primordial conocer la situación del talento intergeneracional en el trabajo con el fin de aplicar una gestión más efectiva que permita mejorar la atracción, retención y colaboración del talento entre las diferentes generaciones. Para ello se precisa investigar sobre cuáles son las diferencias intergeneracionales y cómo se perciben entre sí estas generaciones en el contexto laboral y específicamente con respecto a los líderes.

El segundo estudio, realizado entre los años 2017 y 2018, puede considerarse una continuación del primero. Lleva por título "Liderazgo intergeneracional: análisis de los comportamientos directivos según la generación de los managers y cómo desarrollar su liderazgo para la gestión de equipos intergeneracionales" .

El estudio se planteó dos objetivos principales:

- Conocer la percepción que las diferentes generaciones de empleados tienen sobre el estilo de dirección de su jefe inmediato, según la generación a la que pertenezca.

- Contrastar las diferencias, en el caso de que existiesen, en el modo de gestionar equipos entre las diferentes generaciones de líderes.

En definitiva, se trataba de identificar cómo lideran equipos los managers de las distintas generaciones y comprender cómo gestionan las áreas principales del trabajo en equipo ${ }^{8}$ y el impacto que la gestión de estas áreas tiene en el desarrollo del talento y en el compromiso de sus colaboradores, pertenecientes a distintas generaciones. El objetivo último consistía en averiguar cómo se podría desarrollar un liderazgo que permitiera avanzar en la gestión de equipos intergeneracionales.

En el siguiente gráfico se presentan las diez categorías.

7 Estudio realizado por el Observatorio Generación y Talento en colaboración con la consultoría Tatum y las profesoras investigadoras Rocío Moldes y Fátima Gómez de la Universidad Europea. El enlace al estudio completo puede encontrarse en: <https://www.generacciona.org/wp-content/uploads/2018/07/230618_Estudio_Edici\%C3\%B3n-I_Liderazgo-Intergeneracional_Observatorio-GT-Universidad-Europea.pdf $>$.

8 En el estudio se establecieron diez áreas principales de gestión: valores, rol de equipo, trabajo en equipo, consecución de objetivos, comunicación eficaz, disfrute/clima, formación, oportunidad de desarrollo, reconocimiento y conciliación. La elección de estas áreas responde al hecho de que cada una de ellas permite observar los comportamientos directivos de los diferentes líderes. Estas áreas de gestión constituyen las categorías de análisis a lo largo de la investigación. 
Figura 1. Categorías de análisis del liderazgo intergeneracional

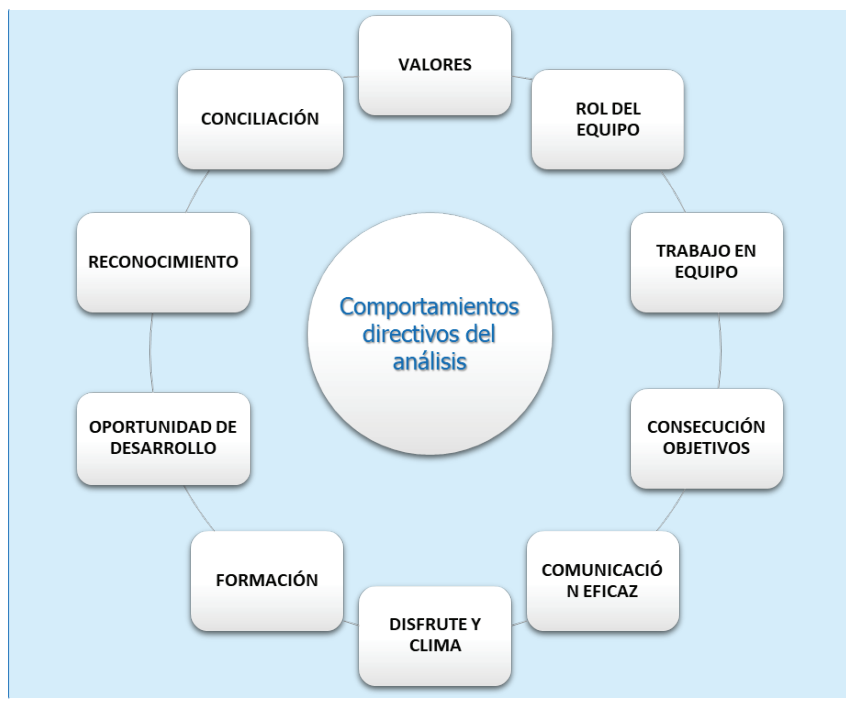

Fuente: elaboración propia. Informe "Liderazgo intergeneracional", Observatorio Generación y Talento \& Rocío Moldes, Fátima Gómez (Universidad Europea, 2018).

La metodología seguida en el estudio fue de carácter cualitativo. Desde el inicio, y dado que se trataba de investigar una realidad poco conocida en el contexto español, se decidió optar por una metodología cualitativa que permitiera captar "los significados particulares que a cada hecho atribuyen sus protagonistas y contemplar estos hechos como piezas de un conjunto sistémico" (Olabuenaga, 2012). Se planteó como un estudio cualitativo y dialógico entre las tres diferentes "voces" protagonistas: directivos, colaborados y profesionales de RR. HH. Se combinaron diferentes métodos de trabajo: por una parte, sesiones de trabajo o workshops en los que participaron conjuntamente managers y profesionales de recursos humanos ${ }^{9}$, y, por otra, grupos de discusión con empleados de diferentes generaciones ${ }^{10}$.

Para recoger la voz de los colaboradores y ahondar en uno de los objetivos planteados —identificar si existen diferentes formas de gestionar equipos en fun-

9 Los workshops se llevaron a cabo por el equipo técnico de la consultoría Tatum (partner técnico del estudio), que utiliza este método de trabajo en sus diferentes estudios sobre análisis de comportamientos en directivos. Las investigadoras Rocío Moldes y Fátima Gómez participaron como observadoras y, en ocasiones, dinamizadoras de las sesiones de trabajo. Esta participación permitió a las investigadoras contextualizar posteriormente, a la hora de analizar los grupos de discusión, las voces de empleados que recogieron en ellos.

10 En el estudio han participado un total de veinticuatro empresas, todas ellas socios colaboradores y miembros de la Red de Empresas del Observatorio GT. Tanto las personas que participaron en los talleres como en los grupos de discusión son empleados de estas organizaciones. Concretamente, en los grupos de discusión participaron cuarenta y seis empleados y empleadas de las empresas colaboradoras en la investigación. Para más información, puede consultarse la página web <https://generacciona.org/estudio-liderazgo-intergeneracional-2/>. 
ción de la generación a la que pertenece el director-, se consideró necesario un enfoque más estructural que permitiese captar y reflejar las relaciones a partir de los discursos. Se optó por la técnica cualitativa del grupo de discusión, dado que mediante el análisis del discurso se pueden captar las relaciones. El grupo de discusión puede definirse como una representación de la realidad, un microcosmos (Ortí, 1986) en condiciones controladas en el que los miembros del grupo colaboran en la definición de sus propios papeles, semidirectivamente orientados por un moderador $^{11}$. Aunque se suele hablar indistintamente de grupos de discusión y focus groups, es preciso matizar que estos últimos tienen un carácter más dirigido y suelen estar orientados a la investigación de mercados. Por su parte, el grupo de discusión es una conversación cuidadosamente planteada, diseñada para obtener información de un área definida de interés, que puede abarcar cualquier ámbito de la vida social, en un ambiente permisivo, no directivo. Los miembros del grupo se influyen mutuamente, puesto que responden a las ideas y comentarios que surgen en la discusión (Krueger, 1991). Así, se programaron grupos de discusión (por cada generación de managers), atendiendo a diferentes criterios. Para diseñar los grupos se decidió como eje estructural la generación a la que pertenecía el manager. Este criterio marcó la homogeneidad de cada grupo. También se tuvo en cuenta un segundo criterio: que en cada grupo estuviese representada la voz de todas las generaciones de empleados que se están analizando en el estudio. Se decidió así trabajar con voces cruzadas. empleados de diferentes generaciones, frente a un eje común: tener un manager de la misma generación.

Durante las sesiones de trabajo los diferentes profesionales fueron relatando sus prácticas y percepciones sobre cada una de las diez áreas de gestión seleccionadas. Así proporcionaban una visión de $360^{\circ}$ capaz de poner de manifiesto las diferentes formas de ejercer el liderazgo atendiendo a la generación del manager y de qué manera impacta en sus colaboradores según su generación. A continuación se presenta una parte de los resultados obtenidos del análisis de los grupos de discusión centrados en la percepción de los empleados sobre los diferentes líderes generacionales en relación con su gestión (clasificada según las categorías de análisis indicadas en el gráfico 1 ).

\footnotetext{
11 El diseño, moderación y análisis de los grupos de discusión fue llevado a cabo íntegramente por las profesoras investigadoras Rocío Moldes y Fátima Gómez, en colaboración con el observatorio Generación y Talento. Se organizaron seis grupos de discusión en los que participaron un total de cuarenta y seis personas. Los grupos se realizaron en el campus de Alcobendas de la Universidad Europea de Madrid entre el 12 y el 21 de septiembre de 2017. La duración media de estos fue de ochenta minutos. Las reuniones fueron grabadas en audio y vídeo para su consiguiente transcripción y análisis con el consentimiento firmado por parte de los participantes. Cabe destacar la dificultad de reunir a empleados de diferentes empresas en horario de trabajo, y más teniendo en cuenta que debían debatir sobre comportamientos de sus managers. No es habitual en el mundo de la empresa que sean los colaboradores quienes opinen sobre sus managers, y menos en un contexto cerrado. El grupo de discusión logró aflorar los discursos en torno al tema planteado, de ahí su riqueza para la comprensión del concepto en estudio: el liderazgo intergeneracional.
} 


\section{a) Generaciones y comportamientos directivos ${ }^{12}$}

Tras el análisis de los discursos obtenidos en los diferentes grupos de discusión, constatamos que las dos generaciones que presentan un mayor desajuste entre autopercepción y percepción de sus colaboradores son baby boomers e Y.

Explicamos a continuación los principales puntos de disenso, apoyados en extractos de los discursos que, a nuestro entender, clarifican el contenido.

- La generación baby boomers: se autoperciben como empleados dedicados, con gran experiencia. Sus colaboradores les definen como controladores y con dificultad para dar instrucciones claras. Las causas de esta fractura entre percepción y autopercepción puede encontrarse en el hecho de que se trata de profesionales que, tras haber iniciado sus carreras en contextos estables y de expansión, se ven obligados a adaptarse a grandes retos en el periodo de madurez de su trayectoria. Tienen tendencia a ver determinados aspectos de la gestión contemporánea como una exigencia del contexto y no como una filosofía en su estilo de dirección.

Existe un salto generacional. Se quiere adaptar a nuevo concepto de mando. Se nota choque entre vieja escuela. Delegar y no delegar según en qué gente. Mientras que mi jefa (X) sí que delega en jóvenes (HGY).

- La generación Y: se autoperciben como empleados orientados a resultados y multitarea. Por su parte, sus colaboradores les consideran cortoplacistas y sobre todo inexpertos en cuanto a la gestión de los equipos, sobre los que trasladan un exceso de presión innecesaria. En opinión de algunos colaboradores:

Crean un clima algo estresante, es la sobrecarga de trabajo que dan al equipo (HGBB).

Otra participante en el grupo de discusión sobre los jefes Y comentaba:

Los managers jóvenes tienen mucha presión de demostrar que valen, y cuando interactúan con otros departamentos quieren coger muchas cosas que trasladan al equipo (MGY).

Las razones de este desajuste se hallan, especialmente, en que los jefes de la generación $\mathrm{Y}$ inician sus carreras en contextos de crisis y en muchas ocasiones no se ha hecho un traspaso adecuado del know how de los jefes pertenecientes a generaciones sénior, baby boomers o X. A pesar de todo, existe cierta unanimidad entre los empleados en percibir a los managers $Y$ como elementos del cambio en las organizaciones. Dentro de un contexto de gran competencia y mucha presión,

12 Se incluyen en este apartado (2.1) y en el siguiente (2.2) comentarios extraídos de los grupos de discusión realizados por las autoras con los empleados de diferentes generaciones. Las siglas utilizadas para su identificación corresponden a: H: hombre; M: mujer; GV: generación veterana; GBB: generación baby boomers; GX: generación X; GY Generación Y. 
se les ofrecen "nuevas oportunidades". Y por su carácter, "ambiciosos y rápidos", encajan en ese nuevo modelo.

En el polo opuesto, las dos generaciones con mayor coincidencia entre autopercepción y percepción de sus colaboradores son los veteranos y la generación $\mathrm{X}$.

La generación de veteranos se autoperciben y se les percibe como eficaces, orientados a resultados y comprometidos y muy alineados con la dirección general de la empresa. Este comportamiento responde a que la mayor parte de su carrera profesional se ha desarrollado en contextos de estabilidad y expansión. Algunos comentarios reflejan la percepción sobre estos veteranos:

No tiene absolutamente nada que demostrar ni a los de arriba ni a los de abajo [...] ya ha llegado al punto en el que los objetivos también le dan igual, ¿vale?, pero aun así te hace ver que tienes tú que terminar de coger las cuatro cosas que estaban un poco más reservadas, para que esto siga funcionando de la misma manera cuando él no este (HGBB).

- Respecto a los jefes X, existe un consenso generalizado en considerar tanto a los empleados como a los managers X una generación "puente". Esta posición de nexo tiene que ver tanto con las grandes transformaciones producidas cuando acceden a posiciones de autoridad — paso del mundo analógico al digital — como con las tendencias contemporáneas de gestión de personas — de organigramas jerárquicos a horizontales - , articulados en torno a la filosofía del liderazgo participativo. Este contexto les ha hecho merecedores del calificativo que, según todos sus colaboradores, les define: flexibles. El comentario de un colaborador respecto a su jefa $X$ ilustra esa sensación:

Se adapta muy bien y controla lo que hace. Dice las cosas claras, pero serenamente (HGV).

Otra de sus características es el ser innovadores en la gestión del equipo. La causa puede buscarse en que deben gestionar dos generaciones muy distintas ("BB", analógica / "Y", digital) y tanto su entrada al mercado laboral como la madurez de sus carreras, en España, se han desarrollado en contextos de crisis.

Como resumen de las percepciones recogidas, se pueden observar en la tabla 1 las características principales que se han ido atribuyendo a cada líder. 
Tabla 1. Percepción del “líder generacional” según la generación del empleado

\begin{tabular}{|c|c|c|c|c|}
\hline \multirow{2}{*}{$\begin{array}{c}\text { EMPLEADOS } \\
\text { POR } \\
\text { GENERACIÓN }\end{array}$} & VETERANOS & $\begin{array}{c}\text { BABY } \\
\text { BOOMERS }\end{array}$ & $\mathrm{X}$ & $\mathrm{Y}$ \\
\cline { 2 - 5 } & Comprometidos & Competitivos & Cualificados & Inexpertos \\
\hline VETERANOS & Eficaces & $\begin{array}{c}\text { Experiencia y } \\
\text { dedicación }\end{array}$ & Buenos gestores & Cortoplacistas \\
\hline BOBY & Rígidos & Controladores & Accesibles & Individualistas \\
\hline $\mathrm{X}$ & $\begin{array}{c}\text { Dificultan } \\
\text { promoción }\end{array}$ & $\begin{array}{c}\text { No instrucciones } \\
\text { claras }\end{array}$ & Ambiciosos & $\begin{array}{c}\text { Soportan } \\
\text { demasiada presión }\end{array}$ \\
\hline
\end{tabular}

Fuente: elaboración propia. Informe "Liderazgo intergeneracional”, Observatorio Generación y Talento \& Universidad Europea, 2018.

La presentación de las percepciones de manera gráfica permita ver, mediante la lectura horizontal, cómo las diferentes generaciones de empleados definen a sus jefes y, mediante una lectura vertical, la caracterización global de cada generación de líderes, lo que posibilita una visión muy completa y apta para su comparación.

\section{b) La gestión del equipo en función de la generación del líder}

Agrupamos en cuatro categorías principales los discursos referidos a cada una de las diez categorías iniciales, con el fin de establecer relaciones explicativas lo más significativas posible en cuanto a los comportamientos de los líderes respecto a su generación de pertenencia:

\section{Dimensión politica de la empresa}

Para hacer operativa la estrategia, es necesaria, entre otras cuestiones, una política empresarial. La empresa es una realidad compleja que exige responder a los retos presentes, con la visión proyectada hacia el futuro. La correcta combinación de las condiciones del contexto, los recursos disponibles y las capacidades que se ponen en juego es lo que hace posible que la visión se convierta en acción eficaz. Los baby boomers aportan experiencia y visión del negocio. Han aprendido a comprender la dimensión política de la empresa, a tener visión estratégica y a orientar los proyectos hacia el valor del largo plazo. Están comprometidos con la empresa, y su responsabilidad y disciplina les hace indispensables en cualquier proyecto. Por su parte, la generación $\mathrm{Y}$ acusa la presión del cortoplacismo, que es el valor de su tiempo; la inmediatez de resultados sustituye en ocasiones a la ausencia de visión estratégica. Uno de los factores que refuerza esta debilidad de las generaciones jóvenes es la inexistencia de modelos de traspaso adecuado del 
know how y de la experiencia de las generaciones anteriores. Estas diferentes concepciones en cuanto al uso del tiempo inciden de forma directa en el clima laboral y la percepción de la calidad del trabajo y el disfrute colectivo.

Controla cosas que nadie sabe, han lidiado con áreas, como los sindicatos... (HGY) (empleados sobre un jefe baby boomers).

\section{Los procesos de comunicación}

Entre las generaciones sénior (veterana y baby boomers) la comunicación es menos frecuente y el feedback se suele utilizar como "forma de corregir" y menos como un modo de "intercambiar puntos de vista". Tienen menos predisposición a la escucha activa entendida como voluntad no solo de escuchar la aportación de los colaboradores, sino de incorporarla a los proyectos.

Entre las generaciones jóvenes ( $\mathrm{X}$ e Y), la comunicación, articulada en torno a un feedback frecuente y personalizado, se convierte en el mecanismo más eficaz para la gestión del equipo. La comunicación, cuando la situación lo requiera, puede tener un carácter informal, y la escucha activa es una constante especialmente para la generación $X$. Véase a este respecto la opinión de un veterano sobre su jefa de la generación $X$ :

Dice las cosas claras, pero serenamente (HGV).

O esta otra empleada sobre su jefe de la generación Y:

Me siento superescuchada, se tienen en cuenta las opiniones (MGY).

La promoción es el resultado de la experiencia, por lo que las exigencias de inmediatez de las generaciones jóvenes (especialmente $\mathrm{Y}$ y $\mathrm{Z}$ ) son un punto de desencuentro. La generación baby boomers facilita el desarrollo de carrera siempre que no lo perciba como una amenaza al propio estatus, con independencia de que sea una oportunidad para el colaborador.

En definitiva, las formas de comunicación han cambiado según las generaciones, percibiéndose más cercanía, escucha y feedback positivo entre las generaciones $\mathrm{X}$ e Y. Esta última generación establece también una comunicación más rápida y digital con sus equipos de trabajo, combinándola con formatos más cercanos e informales.

\section{El trabajo colaborativo}

Con independencia de su generación, todos los managers entienden la importancia contemporánea del equipo; prueba de ello es el desarrollo de organigrama horizontal. Sin embargo, existen diferencias en torno a la capacidad y la forma de delegar. 
Las generaciones sénior suelen tener un planteamiento más normativo que consiste en guiarse por procedimientos establecidos, es decir, las directrices planteadas desde la dirección general de la compañía. Esta práctica se refleja especialmente en la manera en que estas generaciones de líderes gestionan el plan de carrera de sus empleados. Por su parte, las generaciones más veteranas, especialmente baby boomers, consideran que tanto los jefes X como Y utilizan la experiencia de las generaciones sénior para conseguir los propios objetivos y no tanto para promover el desarrollo del empleado.

Las generaciones intermedias, especialmente la $\mathrm{X}$, y las más jóvenes suelen considerarse un punto de inflexión a la hora de delegar responsabilidades y dar autonomía a sus colaboradores. Les cuesta tan poco delegar que en algunas ocasiones los colaboradores se quejan de cierta falta de supervisión. La causa de este modelo de gestión responde al hecho de que la generación $\mathrm{X}$ está entre dos generaciones muy preparadas (baby boomers e Y), $y$, en consecuencia, asumen en gran medida la idea de que los objetivos solo se consiguen con la implicación del equipo. En sintonía con los X, la generación de jefes $\mathrm{Y}$ tiene muy en cuenta el criterio de cada colaborador, algo muy ligado a la idea del team building; en este sentido, los líderes se identifican en la forma de trabajar con equipos de su misma generación. Así se expresaba una colaboradora Y sobre su jefe millennial:

Los tres que formamos el equipo somos muy jóvenes. El trabajo ha sido muy nuevo para los tres, y mi experiencia ha sido la de intentar hacer el trabajo lo mejor posible y aprendiendo. Nos trata como a iguales. Se comparten planteamientos. Se coincide en experiencias. Se gestionan conflictos con dinámicas (MGY).

\section{La conciliación entre vida personal y laboral}

Es, sin duda, el comportamiento en el que se producen las mayores contradicciones entre generaciones.

Las generaciones sénior (especialmente veteranos) muestran una actitud reactiva. No dificultan la implantación de estas políticas, pero, guiados por la lógica que asocia presencia y productividad, no son proactivos.

Entre las generaciones más jóvenes, la $\mathrm{X}$ es la más activa, dado que es la primera generación en la que las mujeres directivas tienen relativa representatividad y sus miembros están en edad de mayores responsabilidades familiares. La generación Y promueve la flexibilidad absoluta y la conexión permanente. Los colaboradores dicen que no tienen una postura clara en este tema y que su compromiso teórico se transforma en real solo cuando comienzan a tener responsabilidades familiares. Así comentaba una colaboradora sobre el cambio de su manager Y al tener hijos: 
Dos de las mangares con las que trabajo han pasado de salir de las 20:30 a las 18:30, ¿y los padres? Se van muy temprano, pero mi jefe no tiene hijos (HGY).

Otra colaboradora comentaba sobre su manager Y:

Mi manager sale tardísimo, pero yo no he tenido problema en salir antes o pedir más flexibilidad (MGY).

En torno a la cuestión de la conciliación, se puede decir que los managers van asumiendo su importancia y son más proclives a establecer y apoyar medidas conciliatorias cuanto más jóvenes son. Sin embargo, se perciben contradicciones entre el discurso y práctica.

\section{c) El "líder ideal"}

Después de analizar en los grupos de discusión los comportamientos de los diferentes directivos por generación, se pidió a los participantes que definieran en tres palabras cuál sería el perfil del líder ideal capaz de potenciar el talento y el compromiso de los equipos independientemente de la generación. En la siguiente tabla se sintetizan las características recogidas.

Tabla 2. Características del líder ideal según sus colaboradores

\begin{tabular}{|c|c|c|c|}
\hline \multicolumn{4}{|c|}{ Principales características que debería tener el "líder ideal" según sus colaboradores } \\
\hline 19 & 20 & 39 & Otras \\
\hline Comunicativo / Comunicador & Comunicativo & Humano & Inteligente \\
\hline $\begin{array}{l}\text { Cercano /Empático, } \\
\text { con Escucha activa }\end{array}$ & Colaborador & Inspirador / Motivador & Participativo \\
\hline Colaborativo & Flexible & Facilitador & Ambicioso \\
\hline Responsable & Innovador & & Capaz de tomar decisiones \\
\hline Integrador & $\begin{array}{c}\text { Adaptativo / Orientado al } \\
\text { cambio }\end{array}$ & & \\
\hline
\end{tabular}

Fuente: elaboración propia. Informe "Liderazgo intergeneracional",

Observatorio Generación y Talento \& Universidad Europea, 2018.

El objetivo de dicha pregunta final fue poder contrastar la "realidad percibida" con la "deseada". En primer lugar, podemos observar que, frente a las diferencias recogidas en torno a los comportamientos de los directivos según las generaciones, se produce un consenso intergeneracional sobre la descripción del "líder ideal". 
Como se refleja en la tabla, existe un acuerdo unánime entre los empleados de las diferentes generaciones en caracterizar al "líder ideal" como una persona comunicativa, cercana, colaborativa, responsable e integradora. Las cualidades más valoradas son las ligadas a la comunicación con un componente más "emocional". Además, se destacan dos características que aluden a la gestión de entornos diversos, como son: que sea innovador y capaz de adaptarse al cambio.

Teniendo en cuenta los resultados obtenidos en el análisis de las percepciones y este perfil de "líder", concluimos que la combinación de diferentes competencias y la adaptación de los comportamientos directivos a diferentes generaciones pueden dar lugar a un nuevo de liderazgo.

Los resultados obtenidos en el análisis permiten afirmar que entender la diferentes personalidades o perfiles dentro de un equipo no solo ayuda a trabajar mejor en conjunto, sino que también contribuye a determinar las fortalezas que le faltan al equipo, mejorando la eficiencia y la productividad. Por ello, aquellas empresas que aprendan a gestionar adecuadamente la diversidad generacional de sus plantillas estarán consiguiendo una ventaja estratégica sobre el resto.

Constatar esta idea a partir de la información analizada nos ha animado a abordar la construcción del liderazgo intergeneracional como un modelo que contribuya a entender el carácter poliédrico de la gestión contemporánea de equipos, caracterizados por su diversidad y altos niveles de cualificación, en el contexto de las tecnologías de la información y la comunicación.

\section{La construcción del liderazgo intergeneracional}

El modelo de liderazgo que denominamos intergeneracional puede ya ir definiéndose como un estilo específico de dirección de personas que, alineado con el liderazgo colaborativo, prioriza el factor generacional combinado con la inteligencia emocional. Este estilo de dirección tiene como primer objetivo lograr una comunicación eficaz, requisito para conseguir el compromiso de los colaboradores.

En la construcción de un modelo es preciso sistematizar aquellos elementos analíticos que nos permitan definirlo. Para ello, tal y como se recoge en la figura 2 , se ha enmarcado cada uno de los comportamientos seleccionados - por entender que abarcan las principales áreas empresariales en la gestión de equipos- entre las dos competencias transversales que, como se ha comprobado, guían el estilo del liderazgo intergeneracional. Se considera que este modelo puede constituirse en la base para ir desarrollando empíricamente y evaluando la efectividad de este liderazgo. 
Figura 2. Modelo de liderazgo intergeneracional

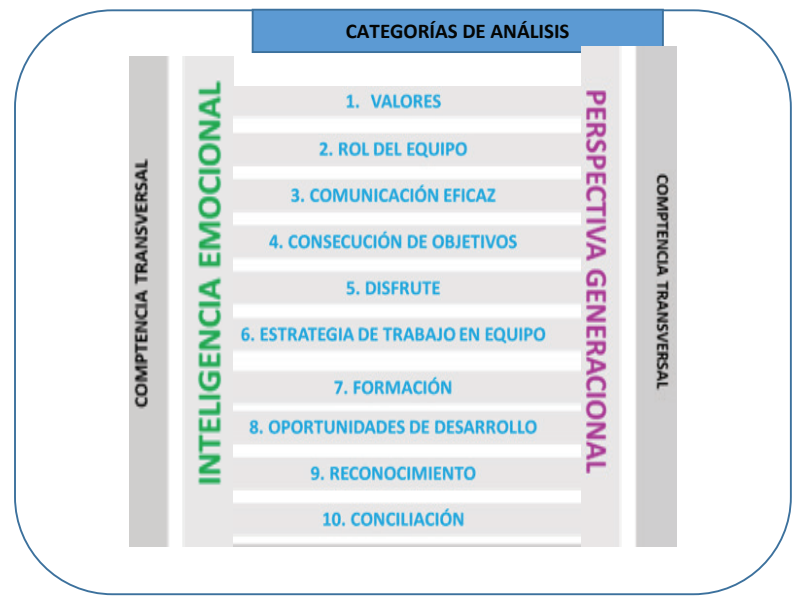

Fuente: elaboración propia. Informe "Liderazgo intergeneracional", Observatorio Generación y Talento \& Universidad Europea, 2018.

A partir de las categorías de análisis seleccionadas, se ha podido comprobar que determinados comportamientos de los líderes son percibidos e impactan de forma diferente en función de la generación tanto del propio líder como del colaborador. De ahí la necesidad de incorporar en la gestión de los líderes la perspectiva intergeneracional, concretándola en una competencia que haría referencia a la capacidad de los managers de comprender y gestionar las diferencias generacionales.

Por otro lado, se ha constatado la necesidad de consolidar las competencias relativas a la inteligencia emocional en la gestión. Habilidades como la comunicación, la empatía, la escucha activa o la capacidad de motivar son vistas como necesarias en un buen líder.

Que los líderes incorporen, por una parte, la inteligencia emocional (Goleman, 2008), definida de forma muy escueta como la capacidad de conectar eficazmente pensamiento y comunicación sobre la base de que nuestras emociones guían (o sesgan) el comportamiento y la toma de decisiones (Damasio, 1994), $y$, por otra, la perspectiva generacional, tal y como se ha planteado a lo largo del trabajo, pone de manifiesto que la habilidad directiva más demandada en el actual contexto es aquella capaz de reconocer y valorar el compromiso.

Una vez revisadas las diferentes competencias que configuran el liderazgo contemporáneo, es preciso dar un paso más y añadir una visión holística de este. Ello nos lleva directamente a la comprensión del liderazgo intergeneracional. Este, además de ser colaborativo, debe ser capaz de ir adaptando las diferentes 
áreas de la dirección (selección, comunicación, evaluación del desempeño, etc.) al modelo del compromiso como requisito para que las organizaciones ejemplifiquen y refuercen el modelo cooperativo y consensuado que las actuales formas de organización social necesitan. La implicación, la colaboración de los empleados, se caracteriza por un alto nivel de versatilidad y un carácter intangible. Solo cuando los empleados están implicados, las empresas pueden retener talento.

Si los responsables de los equipos no logran el compromiso de sus colaboradores, estarán manteniendo plantillas de trabajadores intercambiables. La retención del talento pasa por tener la capacidad de identificar la creatividad de los empleados, como germen para obtener el producto más demandado: ideas originales que tengan valor. Estas ideas originales son, a su vez, el requisito para conseguir el objetivo último de cualquier organización contemporánea: la innovación. Solo mediante una comunicación eficaz, apoyada en grandes dosis de empatía y capacidad para delegar, resistir la presión y adaptarse a la rapidez de los cambios, las empresas son capaces de responder a la competitividad derivada del desplazamiento de las cualificaciones y el auge de las competencias (Boyatzis et al., 2002). En este juego de incentivos, se confirma la importancia de gestionar lo que no se puede medir como base de la dirección de personas contemporánea, frente al aforismo que señala: "Lo que no se puede medir, no se puede gestionar".

Así, el liderazgo intergeneracional se presenta como una dimensión del liderazgo de tipo colaborativo e integrador que, adaptado a las diferentes generaciones, puede sentar las bases para hacer realidad la ventaja competitiva de una gestión adecuada de la diversidad generacional. Las empresas necesitan instrumentos para transferir el conocimiento y, al mismo tiempo, líderes capaces de conocer, atraer y gestionar a las nuevas generaciones, que serán las impulsoras del cambio. Solo cuando aprendamos a gestionar equipos diversos seremos capaces de poner en valor el talento intergeneracional.

\section{Conclusiones}

A lo largo de las dos últimas décadas y a nivel internacional, el interés académico por la gestión de la diversidad generacional constata que la dirección de personas en las organizaciones exige una gestión adaptada a cada segmento de trabajadores para responder a los cambios demográficos y culturales del mercado laboral, con el fin de captar y retener talento.

Existe consenso respecto a la idea de que aquellas empresas que aprendan a gestionar adecuadamente la diversidad generacional de sus plantillas estarán consiguiendo una ventaja estratégica sobre el resto. Cualquier organización que aspire a operar en un mundo global y diverso deberá conocer la situación de la 
diversidad generacional con el fin de aplicar una gestión más efectiva que permita mejorar la atracción, retención y colaboración del talento entre las diferentes generaciones.

En el mercado de trabajo español actual conviven cinco generaciones diferentes caracterizadas según sus estilos de trabajo e intereses personales, cuya gestión no es solo un desafío, sino, sobre todo, un imperativo. Este hecho pone de manifiesto la necesidad de identificar la existencia de diferentes estilos de liderazgo ligados a la generación del líder, así como la relevancia de explorar las expectativas de los empleados respecto a las características del "líder ideal".

Guiados por una metodología cualitativa y desde una perspectiva dialógica entre las tres diferentes "voces" protagonistas - directivos, colaboradores y profesionales de RR. HH.—, los resultados del estudio "Liderazgo intergeneracional: análisis de los comportamientos directivos según la generación de los managers y cómo desarrollar su liderazgo para la gestión de equipos intergeneracionales" pueden sintetizarse en dos ideas. La primera es que, en efecto, existen diferentes modos de gestión en función de la generación a la que pertenezca el jefe. La segunda constata que lo que más valoran los empleados, con independencia de su generación, es la capacidad de comunicación de sus managers.

De los diez comportamientos seleccionados como categorías de análisis, las principales diferencias en la forma de gestión de los jefes, atendiendo a su generación, se concentran en cuatro aspectos:

a. La dimensión política de la empresa (visión estratégica y orientación temporal de los proyectos)

b. Los procesos comunicativos (formas de feedback, frecuencia y concepción de este)

c. El trabajo colaborativo (papel del equipo, capacidad para delegar)

d. La conciliación entre vida profesional y personal (gestión del tiempo, concepción del trabajo flexible)

A la luz de los resultados obtenidos en el estudio, proponemos un modelo en el que el liderazgo intergeneracional esté enmarcado en dos competencias transversales. Por una parte, la perspectiva intergeneracional, concretándola en una competencia que haría referencia a la capacidad de los managers de comprender y gestionar las diferencias generacionales. Por otra, la inteligencia emocional en la gestión, entendida como la capacidad de conectar eficazmente pensamiento y comunicación sobre la base de que nuestras emociones guían (o sesgan) el comportamiento y la toma de decisiones. 
Con el fin de lograr una visión holística del modelo propuesto, el liderazgo intergeneracional puede definirse como un estilo específico de dirección de personas que, alineado con el liderazgo colaborativo, prioriza el factor generacional combinado con la inteligencia emocional para conseguir una comunicación eficaz, entendida como la principal herramienta para lograr el compromiso de los colaboradores.

En definitiva, el modelo del liderazgo generacional puede considerarse un primer paso en el diseño de un marco de gestión y evaluación de competencias capaz de rentabilizar la ventaja competitiva de la diversidad generacional.

\section{Referencias bibliográficas}

Alonso Puig, M. (2004). Madera de líder. Claves para el desarrollo de las capacidades de liderazgo. España: Empresa Activa.

Allen, N.J.y Meyer, J.P. (1990). "The measurement and antecedents of affective, continuance and normative commitment to the organization". Journal of Occupational and Organizational Psychology, 63(1), 11-18.

Álvarez De Mon, S. (2009). El mito del líder. España: Prentice-Hall.

Ambler, T. y Barrow, S. (1996). “The employer Brand”. Journal of Brand management, 483 (pp. 185-2016).

Boyatzis, R.; Goleman, D. y McKee, A. (2002). El líder resonante crea más. El poder de la inteligencia emocional. Barcelona, España: Plaza \& Janes Editores.

Caraher, L. (2017). Millennials en la oficina. Cómo lidiar con una generación que no sigue las reglas. México, Paidós.

Cheese, P. et alii (2008). La organización basada en el talento. Madrid: Pearson Education.

Confederación Española de Directivos y Ejecutivos CEDE \& Repsol (2015). Informe: "La diversidad en la gestión directiva".

Conger, J. (1993). Learning to lead the art of transforming managers into leaders. San Francisco: CA. Jossey-Bass.

Damasio, A. (1994). El error de Descartes. Emoción, razón y cerebro bumano. Barcelona: Planeta.

Drucker, Peter F. (1996). La gestión en un tiempo de grandes cambios. Argentina: Editora y Distribuidora Hispano Americana.

Dychtwald, K.; Erickson, T. y Morison, R. (2007). Retención del talento. Madrid: LID Editorial empresarial, S. L.

Gavilán, D.; Avelló, M.y Fernández-Lores, S. (2013)."Employer branding, la experiencia social de la marca y sus efectos sobre el compromiso afectivo". DeResearch ESIC, $\mathrm{n}^{\circ} 7$, volumen 7. 
Gavilán, D;; Avelló, M.; Fernández-Lores, S. y Andrés E. (2014). "Medir el Compromiso, el nuevo Must de RR. HH.". Observatorio de recursos bumanos y relaciones laborales, $\mathrm{n}^{\circ} .95$ (pp. 32-39).

Gilbert, G. (2011). "Los Millennials: una nueva generación de empleados, un nuevo conjunto de políticas de compromiso". Ivey Business Journal. Recuperado de: https://iveybusinessjournal.com/publication/the-millennials-a-newgeneration-of-employees-a-new-set-of-engagement-policies/

Goleman, D. y Drucker, F. (2018). Liderando personas. Barcelona: Profit editorial.

Goleman, D. (1995). Emotional intelligence. Nueva York: Bantam books.

Gómez Sota, F• y Cordero, R. (2009). "La gestión del talento en la empresa del siglo XXI”. Revista Gaudeamus. Revista Académica de la Universidad Interamericana de Costa Rica. Año $1, \mathrm{n}^{\circ} 1$.

Harvard Business Review (2016). The Harvard Business Review Manager's Handbook: The 17 Skills Leaders Need to Stand Out. Harvard Business Review Press.

Huertas, A. y Ortega, I. (2018). La revolución de las canas. Ageingnomics o las oportunidades de una economía del envejecimiento. Barcelona: Gestión 2000.

IвÁÑez, J. (1979). Más allá de la sociología. El Grupo de discusión: técnica y crítica. Madrid: Siglo XXI

Kanter, R. M. (1968). "Commitment and social organization. A study of commitment mechanisms in utopian communities". American Sociological Review, 33 (pp. 499-517).

Krueger, R. A. (1988). El grupo de discusión. Guía Práctica para la investigación aplicada. Madrid: Pirámide.

Las Heras, M. y Jiménez, E. (2012). “Generaciones y Talento: 2012”. IESE Business School Universidad de Navarra, RRHH 365.

Maella, P. (2008). Gestionar con sencillez: Mitos y realidades del liderazgo. Barcelona: Profit.

MArí, P. (2017). Liderar equipos comprometidos. Madrid: Plataforma.

Moldes Farelo, R. (2012). De la gestión de recursos bumanos a la dirección de personas. Valencia: Tirant lo Blanch.

Moldes Farelo, R. (2010). "El desarrollo del liderazgo competitivo como estrategia en las organizaciones de vanguardia". Trend Management, vol. 12, no 6 (pp. 1-5).

Olabuenaga Ruiz, J. (2012). Metodología de la investigación cualitativa (5a ed.). Bilbao: Universidad de Deusto. 
Ortí, A. (1986). "La apertura y el enfoque cualitativo o estructural: la entrevista abierta, semidirectiva y la discusión de grupo" (153-486). En M. GArcía FERRANDo et alii. El análisis de la realidad social. Madrid: Alianza Universidad.

PAstor, J. (2009). "El liderazgo de personas en las organizaciones". En J. BonAche y A. Cabrera (directores). Dirección de personas. Evidencias y perspectivas para el siglo XXI. Madrid: Pearson.

Prensky, M. (2001). Digital Natives, Digital Immigrants. Recuperado de: <http://marcprensky.com/>.

Redondo-Sama, G. (2020). "Supporting Democracy Through Leadership in Organizations”. Qualitative Inquiry, 26(8-9), 1033-1040. Recuperado de: <https://doi.org/10.1177/1077800420938885>.

Salmon, C. (2009). Storytelling: la máquina de fabricar historias y formatear las mentes. Barcelona: Península.

Universum (2017). "Building leaders for the next decade". Informe ejecutivo. Recuperado de: <https://universumglobal.com/building-leaders-next-decade $/>$.

Warner, J. y León, C. (2010). Liderazgo intergeneracional. Madrid: Ramón Areces.

Winnen, L. (2015). Human capital and organizational performance: a causal model. Tesis doctoral. Universidad Europea de Madrid (Depósito biblioteca CRAI).

Woolfe, L. (2004). Valores para lideres contemporáneos: Lecciones de administración y liderazgo. Madrid: Cecsa. 